Abstract PS4:82 Table 1 General and SLE-correlated risk factors in 'APO' and 'without APO' groups

\begin{tabular}{|c|c|c|c|}
\hline General risk factors & APO $\%$ & Without APO \% & $P$ value \\
\hline Age $>35$ years & 35,3 & 30,5 & 0,491 \\
\hline Hypertension & 17,6 & 16,1 & 0,722 \\
\hline Diabetes mellitus & 0,0 & 0,8 & 1,000 \\
\hline Obesitiy & 11,7 & 5,9 & 0,712 \\
\hline Thyroid disease & 0,0 & 5,9 & 1,000 \\
\hline Cigarettes smoking & 41,2 & 20,3 & 0,187 \\
\hline SLEDAI $>0$ & 86,7 & 83,9 & 1,000 \\
\hline SLEDAI >6 & 14,3 & 14,8 & 1,000 \\
\hline dsDNA & 84,6 & 62,6 & 0,172 \\
\hline Lupus nephritis & 52,9 & 33,0 & 0,167 \\
\hline Low C3 and/or C4 & 50,0 & 54,6 & 0,790 \\
\hline Ro and/or La & 53,8 & 41,9 & 1,000 \\
\hline $\mathrm{aPL}$ & 64,6 & 51,6 & 0,278 \\
\hline Triple aPL & 23,5 & 11,8 & 0,244 \\
\hline LAC & 23,5 & 21,2 & 0,760 \\
\hline Previous thrombosis & 0,0 & 3,4 & 1,000 \\
\hline Previous APO * & 35,3 & 21,2 & 0,221 \\
\hline
\end{tabular}

${ }^{*} \mathrm{APO}$ were defined as premature miscarriage $\left(<10^{\wedge}\right.$ weeks), fetal death $\left(>10^{\wedge}\right.$ weeks), preterm delivery $\left(<34^{\wedge}\right.$ weeks) with or without preeclampsia, HELLP Syndrome, perinatal death ( $<30^{\wedge}$ day).

1. by comparing the outcome of prospectively-followed pregnancies (PFP) and anamnestic pregnancies (AP);

2. by comparing the outcome of PFP with the general obstetric population (GOP);

3. by evaluating the disease features, maternal risk factors and treatment of pregnancies with adverse pregnancy outcome (APO) in PFP.

Methods A monocentric, retrospective and observational study of 94 SLE patients with a total of 135 pregnancies followed prospectively by multidisciplinary team. In addition, $33 \mathrm{AP}$ in the same SLE patients and 3939 pregnancies among GOP were evaluated. Clinical and serological data were obtained from medical records.

Results The comparison between PFP and AP showed lower frequency of premature miscarriage $(6,7 \%$ vs $27,3 \%$, p value $0,0021)$ and fetal death $(3,7 \%$ vs $36,4 \%$, p value $<0,0001)$ and higher frequency of live birth $(88,9 \%$ vs $36,4 \%$, p value $<0,0001)$ in the first group. As compared with GOP, SLE-PFP displayed similar rate of early miscarriage $(9,0 \%$ vs $6,7 \%)$ and fetal loss $(5,0 \%$ vs $3,7 \%)$ but higher frequency of preeclampsia $(1,0 \%$ vs $5,0 \%$, p value 0,0029$)$, preterm birth $(7,0 \%$ vs $18,4 \%, \mathrm{p}$ value $<0,0001)$ and Caesarian section $(31,0 \%$ vs $41,7 \%$, p value 0,0288$)$.

APO occurred in $17(12.6 \%)$ of the 135 PFP. Despite the lack of statistical significance, there was a tendency toward higher frequency of anti-dsDNA positivity (84,6\% vs 62,6\%), history of lupus nephritis $(52,9 \%$ vs $33,0 \%)$ and triple antiphospholipid antibody (aPL) positivity $(23,5 \%$ vs $11,8 \%)$ in pregnancies with APO (table 1). Analysing treatment during pregnancy, the group with APO received higher doses of prednisone (without significant $\mathrm{p}$ value) and required higher use of immunosuppressants $(64,7 \%$ vs $31,3 \%$, p value 0,032$)$.

Conclusions The outcome of PFP in SLE has dramatically improved as compared to AP, thanks to pregnancy planning, multidisciplinary management and close monitoring during pregnancy. The occurrence of APO was restricted to a minority of PFP $(12,6 \%)$. SLE-PFP had similar rates of pregnancy losses as compared to GOP, but there are still open issues on some pregnancy complications that affect SLE patients more frequently.

\section{PS4:83 FOLLOW-UP OF NEWBORN BABIES FROM MOTHER AFFECTED BY SYSTEMIC AUTOIMMUNE DISEASE}

${ }^{1}$ Tiziana Bertero, ${ }^{2}$ Jessica Munarin, ${ }^{2}$ Mario Frigerio, ${ }^{2}$ Emilia Parodi. ${ }^{1} /$ mmunology Unit, ASO Mauriziano Hospital, Torino, Italy; ${ }^{2}$ Pediatric and Neonatology Unit, ASO Mauriziano Hospital, Torino, Italy

\subsection{6/lupus-2018-abstract.128}

Introduction Most autoimmune systemic diseases affect more frequently females in reproductive age, suggesting the need to consider the possible effect on some important aspects of women's life such as fertility and pregnancy, but above all possible outcomes on the newborn and, subsequently, on the child.

Topic of this study is the analysis of the neonatal and long-term paediatric outcomes, until school age, of a group of paediatric subjects born from mother affected by systemic autoimmune disease, in care at the Department of Neonatology and Paediatrics of the Mauriziano Hospital of Turin, in order to identify any associations with pregnancy and the type and activity of maternal disease.

Materials and methods From October 2016 all women in care at the Department of Immunology of the Mauriziano Hospital, aged between 25 and 45, who had one or more pregnancies hesitated in the birth of alive baby during January 2002 and October 2015, have been enrolled in this study.

We have considered, for each pregnancy $(n=48)$ : type of maternal disease (with antibody dosage), obstetric outcomes (type of delivery, indication to caesarian section, gestational age at birth), neonatal outcomes (Apgar score, low neonatal weight, fetal growth restriction, neonatal complications), clinical-diagnostic management of babies (hematologic and other examinations required) and long-term paediatric outcomes (psychomotor development alterations, chronic diseases, hematologic alterations, hospital admissions). 
Results In our study we have observed a greater incidence of preterm birth (33\%) and caesarian section (68\%), compared to reference healthy population of the same hospital Department. We also observed, in association with maternal therapy taken during pregnancy, a significant difference of the incidence of preterm birth $(p=0,0005)$ and a weakly significant difference of the incidence of low neonatal weight $(\mathrm{p}=0,0596)$.

We haven't notice any significant difference, in association with antibody positivity, of the incidence of low birth weight and fetal growht restriction and not even in the incidence of neoantal complications.

Regarding the long-term paediatric follow-up, we have notice a greater incidence of psycomotor development alterations in association with ENA antibody positivity $(28 \%$ vs $0 \%)$, with a statistically significant difference $(p=0.0155)$.

Conclusions Data from the retrospective analysis, in agreement with literature, confirm a greater incidence of caesarian section (most of cases programmed), preterm birth and low birth weight in this category of newborns.

For the first time it was considered the association between neonatal and paediatric outcomes and the maternal tharapy during pregnancy: we have notice a significant greater incidence of preterm birth in newborns from mother who have taken therapy during pregnancy.

Regarding the long-term paediatric follow-up, until school age, in agreement with the few data in the literature, it was observed a greater incidence of psychomotor development alterations in children born from mother with antibody positivity (aPL ed ENA).

Our data notice a poor adherence to practical recommendations of the clinical management and the follow-up of these children present in the literature.

We have set up a national survey in order to verify current knowledge and to sensitise Paediatricians and Neonatologists regarding this category of children, that need a specific clinical management and follow-up.

Since limits of a retrospecive study and the relative small population size, we started a case-control prospective study with a wider population size, currently in the recruitment phase, in order to validate data observed in the retrospective analysis and to verify the current adhesion to raccomandations of the follow-up.

We hope that a greater knowledge of the topic, together with the establishment of common clinical practices, can bring to an improvement of obstetric, neonatal and long-term paediatric outcomes in children born from mother with systemic autoimmune disease.

\section{PS4:84 OBSTETRICAL COMPLICATIONS AND PREGNANCIES IN WOMEN WITH SYSTEMIC LUPUS ERYTHEMATOSUS}

T Ben Salem, M Tougorti, I Naceur, M Lamloum, I Ben Ghorbel, MH Houman. Department of Internal Medicine, La Rabta University Hospital, Tunis, Tunisia

\subsection{6/lupus-2018-abstract.129}

Background Systemic lupus erythematosus (SLE) is a chronic auto-immune disease, which mainly occurs in women during childbearing age. It is associated with several fetal and maternal complications.

Objective To describe obstetrical complications and evaluate pregnancy and fecundity rates in women with SLE.
Methods It is a retrospective study including 227 women with SLE. Only 143 women, who are sexually active, were studied. Results The mean age at disease onset was $36.61 \pm 13.41$ years. The mean age at SLE diagnosis was $38.47 \pm 13.43$ years. The most frequent manifestations of the disease were lupus nephritis and neurological involvements, seen in respectively $39.7 \%$ and $14.7 \%$ of patients. Antinuclear antibodies were positive in $96.5 \%$ of cases and anti-dsDNA were positive in $96.3 \%$ of cases. Anti-SSA, anti-SSB, anti-cardiolipin and anti-B2GPI antibodies were found in respectively $68,37.3,46 \%$ and $27.3 \%$ of patients. Corticosteroids were given in $90.4 \%$ of patients and 58\% of them had immunosuppressive therapy: Cyclophosphamide $(n=42)$, Azathioprine $(n=30)$, Methotrexate $(n=9)$ and Mofetil Mycophenolate $(n=2)$.

Obstetrical complications were: spontaneous abortion before the $10 \mathrm{TH}$ week of gestation (22.8\%), unexplained fetus death after the $10 \mathrm{TH}$ week of gestation (12.2\%) and intrauterine death (10\%).

Among the 143 women, 130 had at least one pregnancy (average $=3.59 \pm 2.62$ pregnancies). Most pregnancies resulted in live births; 121 women had at least one child with an average of $2.55 \pm 2$ children/woman. Pregnancies occurred after SLE diagnosis in 40 women (among 69 who wants to have children) with a mean of $1.75 \pm 1.05$ pregnancies. Twenty nine women had at least one child with a mean of $1.15 \pm 0.9$ children. In addition to their usual treatment, other drugs were added during pregnancies: aspirine $(n=7)$, low molecular weight heparin $(n=7)$ and betamethasone in one case. One patient was treated with aspirine and low molecular weight heparin. Obstetrical events caused lupus flare in 11 cases (pregnancy in 7 cases, delivery in 2 cases, intrauterine death and spontaneous abortion each in one case).

Conclusion Comparing to general population in Tunisia (2 children/woman), fecundity is reduced in women after SLE diagnosis. Complications are related to disease activity, antiphophspholipid antibodies and hypertension.

\section{PS4:85 FERTILITY AND PREGNANCY IN WOMEN WITH SYSTEMIC LUPUS ERYTHEMATOSUS: A COMPARISON WITH OTHER RHEUMATIC DISEASES}

${ }^{1}$ IC Chalmeta-Verdejo, ${ }^{1} \mathrm{~K}$ Arevalo, ${ }^{1} \mathrm{~J}$ Ivorra, ${ }^{2} \mathrm{C}$ Diaz-García, ${ }^{1} \mathrm{R}$ Negueroles, ${ }^{1} \mathrm{E}$ Grau, ${ }^{1} \mathrm{~L}$ Gonzalez-Puig, ${ }^{1}$ I Martinez-Cordellat, ${ }^{1} \mathrm{C}$ Feced, ${ }^{1} \mathrm{~F}$ Ortiz, ${ }^{1} \mathrm{C}$ Alcañiz, ${ }^{1} \mathrm{E}$ Vicens, ${ }^{1} \mathrm{~J}$ Oller, ${ }^{1} E$ Labrador, ${ }^{1} \mathrm{~J}$ Fragio, ${ }^{1} \mathrm{R}$ González, ${ }^{1} \mathrm{M}$ de la Rubia, ${ }^{1} \mathrm{C}$ Nájera, ${ }^{1} \mathrm{I}$ Canovas, ${ }^{1} \mathrm{~J}$ A RománIvorra. ${ }^{1}$ Rheumatology Department. Hospital Universitario la fe, Valencia, Spain; ${ }^{2}$ Unidad de Preservacion de Fertilidad, Hospital Universitario la fe, Valencia, Spain

\subsection{6/lupus-2018-abstract.130}

Purpouse Rheumatological disorders, usually affect women during reproductive ages and have an impact on their reproductive wishes.

We aim to assess the differences between SLE and other diseases for fertility and pregnancy evolution in a group of patients followed in a specific consult for planning and management of pregnancy.

Methods Retrospective study in a cohort of patients with rheumatological disorders who want to get pregnant. Fertility exam to the couple was done. Data about sterility (never conception) and infertility (miscarriages), assisted reproduction, preterm delivery and also adverse pregnancy outcomes (disease flares, preeclampsia) were compared in 3 groups: SLE, connective tissue disease non-SLE (Sjögren's Syndrome, MCTD) and 